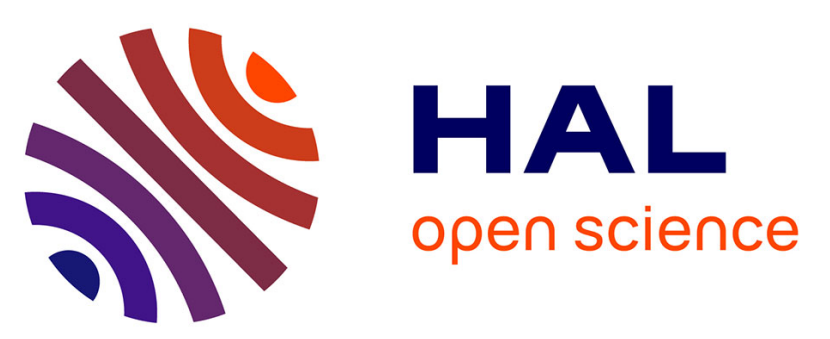

\title{
LES BÉBÉS PRÉMATURÉS PERÇOIVENT-ILS LA SYNCHRONIE AUDIO-VISUELLE À 4 MOIS? CONTRIBUTION DE L'ANALYSE OCULOMÉTRIQUE
}

Françoise Morange-Majoux, Christelle Lemoine-Lardennois, Éric Orriols, Joëlle Provasi

\section{To cite this version:}

Françoise Morange-Majoux, Christelle Lemoine-Lardennois, Éric Orriols, Joëlle Provasi. LES BÉBÉS PRÉMATURÉS PERÇOIVENT-ILS LA SYNCHRONIE AUDIO-VISUELLE À 4 MOIS? CONTRIBUTION DE L'ANALYSE OCULOMÉTRIQUE. Enfance, 2017, 10.4074/S001375451700413X . hal02887729

\section{HAL Id: hal-02887729 \\ https://hal.science/hal-02887729}

Submitted on 2 Jul 2020

HAL is a multi-disciplinary open access archive for the deposit and dissemination of scientific research documents, whether they are published or not. The documents may come from teaching and research institutions in France or abroad, or from public or private research centers.
L'archive ouverte pluridisciplinaire HAL, est destinée au dépôt et à la diffusion de documents scientifiques de niveau recherche, publiés ou non, émanant des établissements d'enseignement et de recherche français ou étrangers, des laboratoires publics ou privés. 


\section{LES BÉBÉS PRÉMATURÉS PERÇOIVENT-ILS LA SYNCHRONIE AUDIO-VISUELLE À 4 MOIS ? CONTRIBUTION DE L'ANALYSE OCULOMÉTRIQUE}

Françoise Morange-Majoux, Christelle Lemoine-Lardennois, Éric Orriols, Joëlle Provasi

NecPlus | «nfance»

$2017 / 4 \mathrm{~N}^{\circ} 4$ | pages 495 à 501

ISSN 0013-7545

Article disponible en ligne à l'adresse :

https://www.cairn.info/revue-enfance2-2017-4-page-495.htm

\section{Pour citer cet article :}

Françoise Morange-Majoux et al., « Les bébés prématurés perçoivent-ils la synchronie audio-visuelle à 4 mois ? Contribution de l'analyse oculométrique », Enfance 2017/4 (N 4), p. 495-501.

DOI 10.4074/S001375451700413X

Distribution électronique Cairn.info pour NecPlus.

(C) NecPlus. Tous droits réservés pour tous pays.

La reproduction ou représentation de cet article, notamment par photocopie, n'est autorisée que dans les limites des conditions générales d'utilisation du site ou, le cas échéant, des conditions générales de la licence souscrite par votre établissement. Toute autre reproduction ou représentation, en tout ou partie, sous quelque forme et de quelque manière que ce soit, est interdite sauf accord préalable et écrit de l'éditeur, en dehors des cas prévus par la législation en vigueur en France. Il est précisé que son stockage dans une base de données est également interdit. 


\title{
Les bébés prématurés perçoivent-ils la synchronie audio-visuelle à 4 mois ? Contribution de l'analyse oculométrique
}

\author{
Françoise MORANGE-MAJOUX ${ }^{1}$, \\ Christelle LEMOINE-LARDENNOIS ${ }^{2}$, Éric ORRIOLS ${ }^{3}$ \\ et Joëlle PROVAsi ${ }^{4}$
}

\begin{abstract}
RÉSUMÉ
L'objectif de cette recherche était d'examiner la perception d'événements audio visuels désynchronisés chez les nourrissons prématurés et nés à terme, en comparant non seulement les temps de regard entre des situations synchro- et désynchronisées mais également en analysant l'exploration visuelle au moyen d'un traqueur oculaire, afin de déterminer si des zones étaient préférentiellement examinées. Dix-huit bébés nés entre 34 et 36 semaines d’Âge gestationnel (AG), douze bébés nés entre 31 et 33 semaines $A G$ et trente-six bébés nés à terme ont été observés à 4 mois. Après avoir été habitués à une scène audio-visuelle synchronisée (cible bougeant verticalement avec un son associé à son rebond), les bébés étaient soumis à une phase test au cours de laquelle leur étaient présentées alternativement 3 fois, une scène désynchronisée (son décalé par rapport au rebond de la cible) et la scène synchronisée. Les résultats montrent que les bébés regardent plus longtemps la scène désynchronisée que le dernier essai d'habituation quel que soit l'âge gestationnel. L'examen de leur exploration visuelle révèle qu'ils regardent plus longtemps la zone de la cible où le son décalé est entendu. Toutefois les bébés nés prématurément regardent moins longtemps la cible que les bébés nés à terme et prennent plus de temps pour s'habituer. Ces résultats sont discutés en termes d'hypervigilance liée à l'expérience extra-utérine acquise par les bébés nés prématurés.

MOTS-CLÉS : ENFANTS PRÉMATURÉS, DÉSYNCHRONISATION TEMPORELLE, SYNCHRONISATION AUDIO-VISUELLE, HYPERVIGILANCE
\end{abstract}

1 Laboratoire de psychopathologie et psychologie de la santé, Université Paris-Descartes, Institut de Psychologie, 71 avenue Édouard Vaillant, 92774 Boulogne Billancourt. Email : francoise.morange-majoux@parisdescartes.fr

2 Laboratoire Vision Action Cognition - EA7326, Université Paris Descartes, Centre Henri Piéron, UFR Institut de Psychologie, 71 avenue Edouard Vaillant, 92774 Boulogne-Billancourt Cedex.Email: christelle.lemoine@parisdescartes.fr

3 LMC, Université Paris Descartes - Inserm - Université Paris Descartes, Centre Henri Piéron, UFR Institut de Psychologie, 71 avenue Édouard Vaillant, 92774 Boulogne-Billancourt Cedex.Email: eric.orriols@parisdescartes.fr

4 Laboratoire CHArt - EPHE, 4-14 rue Ferrus, 75014 Paris. E-mail : joelle.provasi@ ephe.sorbonne.fr 


\section{ABSTRACT \\ Perception of auditivo-visual synchrony in preterm infants: Contribution of analysis with the eye-tracking system at 4 months}

The aim of this study was to investigate auditory-visual temporal asynchrony in preterm infants using a habituation procedure coupled with an eye-tracking system in order to examine visual behavior accurately and determine specific visual areas of interest. Infants were habituated with an auditory-visual synchronic situation: a visual ball bounced back in synchrony with an auditory sound. In the test phase, an asynchronized situation and a synchronized situation were presented alternately three times. Eighteen infants, born between 34 and 36 weeks of gestational age, twelve infants, born between 31 and 33 weeks GA and thirty-six full-term infants were tested at four months post term. The results showed that babies looked at the desynchronized scene longer than the last habituation trial, whatever gestational age. The study of areas of interest revealed that whatever the age group, infants looked more at the area where the sound was produced during the asynchronized trial. However, pre-term infants spent less time on the target than full-term infants and had a more heterogeneous visual exploration. The results are discussed in terms of hyper vigilance linked to ectopic experience acquired by preterm babies.

KEY-WORDS: PRETERM INFANTS, TEMPORAL ASYNCHRONY, AUDITORY-VISUAL ASYNCHRONY, HYPER-VIGILANCE 


\section{INTRODUCTION}

La perception des aspects temporels de l'environnement joue un rôle essentiel dans le développement sensori-moteur, social, émotionnel et cognitif du jeune enfant. Dès la naissance, le nouveau-né est sensible à la contingence entre 2 événements séparés dans le temps, comme par exemple les actes de communication (Feldman \& Eidelman, 2007). À 4 mois et demi, il est capable de percevoir les correspondances rythmiques entre les événements auditifs et visuels, comme une balle qui rebondit en faisant un bruit à chaque rebond (Lewkowicz, 1992).

L’enfant prématuré est plongé dans un environnement fortement chargé en stimulations sensorielles variées, pouvant être à la fois non contingentes et sans relation de cause à effet, l'empêchant d'avoir une perception unitaire de l'information. Les performances cognitives sont généralement moins bonnes (Mellier, 2017), en particulier, on note un retard dans la perception de la synchronie. Ainsi les enfants prématurés ne perçoivent pas la désynchronisation temporelle entre un visage et sa voix (Pickens et al., 1994).

Pour autant, ces enfants bénéficient d'une expérience additionnelle extrautérine, notamment d'une exposition plus précoce au monde visuel (Bloch, Lequien, \& Provasi, 2003). Certains auteurs notent des réponses visuelles mieux organisées chez les prématurés par rapport aux enfants nés à terme et de bonnes capacités d'habituation (Mellier, 2017). Ils sont également plus rapides à se désengager visuellement et à modifier leur attention du champ visuel central au champ périphérique. Ils semblent donc plus actifs que réactifs, peut-être en lien avec un état d'alerte qu'on pourrait assimiler à un état d'hypervigilance (Bullinger \& Goubet, 1999).

L'objectif de cette recherche est d'étudier la perception d'événements audio visuels désynchronisés chez les nourrissons prématurés et nés à terme, en comparant non seulement les temps de regard entre des situations synchro- et désynchronisés mais également en analysant finement l'exploration visuelle au moyen d'un traqueur oculaire.

\section{MÉTHODE}

\section{Sujets}

Trente bébés prématurés (12 filles) ont été observés à 4 mois d’âge corrigé. Dix-huit d'entre eux étaient nés entre 34 et 36 semaines d'âge gestationnel (AG) (poids moyen $=2066 \mathrm{~g}$ ) et douze entre 31 et 33 d'âge gestationnel (poids moyen $=1837 \mathrm{~g}$ ). Tous étaient en bonne santé et ne présentaient aucune complication liée à leur prématurité, ni aucun trouble visuel. Trente-six bébés nés à terme (20 filles) ont été également observés à 4 mois (poids moyen $=3120 \mathrm{~g}$ ).

\section{Matériel}

Le matériel présenté aux enfants se composait de deux films présentant une balle verte montant et descendant au centre de l'écran $(0,19 \mathrm{~m} / \mathrm{sec})$. Dans la première 
vidéo, un son était associé au rebond de la balle en bas de l'écran, scène appelée synchronie audio-visuelle; dans la seconde vidéo, le son était décalé de $450 \mathrm{~ms}$ après le rebond de la balle en bas de l'écran, scène appelée désynchronie audiovisuelle. Un système de traqueur oculaire (TOBII 1750) a été utilisé.

\section{Procédure}

Les bébés étaient installés dans une pièce calme, sur les genoux d'un parent, face à un écran, à une distance de $70-73 \mathrm{~cm}$. Après une phase de calibration nécessaire pour enregistrer le regard de chaque bébé, la vidéo synchronisée était diffusée (phase d'habituation) : la balle verte apparaissait en haut de l'écran et commençait à bouger vers le bas, le bébé la suivant des yeux. Dès que les yeux du bébé sortaient de l'écran plus d'une seconde, la vidéo s'arrêtait et un nouvel essai débutait. Entre chaque essai, un clown apparaissait au centre de l'écran afin d'attirer le regard de l'enfant. La vidéo synchronisée pouvait être présentée jusqu'à 15 fois, afin d'atteindre le critère habituation (temps moyen de 3 essais consécutifs moitié moins longs que le temps moyen des 3 premiers essais). Après la phase d'habituation, la phase test consistait à présenter alternativement 3 fois, les vidéos désynchronisée et synchronisée.

\section{Analyses}

Le temps total passé sur l'écran et le nombre d'essais pour atteindre le critère d'habituation ont été analysés avec le logiciel E-Prime. La distribution spatiale des points de regard sur l'écran a été enregistrée par le système de traqueur oculaire. Plusieurs zones d'intérêt ont été définies (fig. 2), notamment la zone où le son décalé est entendu dans la scène désynchronisée, son exploration visuelle plus active pouvant signer que les bébés ont détecté la désynchronie. Des analyses statistiques (ANOVAs) ont été effectuées.

On s'attend à ce que les bébés prématurés s'habituent moins vite et soient moins nombreux que les bébés nés à terme. Les bébés prématurés devraient également avoir des difficultés à percevoir la synchronie et leur regard devrait être plus dispersé sur l'écran que celui des bébés nés à terme.

\section{RÉSULTATS}

\section{Habituation}

$67 \%$ des bébés prématurés $(n=20)$ et $44 \%$ des bébés nés à terme $(n=16)$ se sont habitués à la scène synchronisée. Le temps moyen pour atteindre le critère d'habituation est plus long chez les bébés de 31-33 sem AG que chez les autres bébés $(\mathrm{F}(2,33)=3,95$; post-hoc Tukey, $p<0,05)$.

\section{Réaction à la nouveauté (fig. 1)}

Les résultats montrent que les bébés, quel que soit le groupe d’âge, passent plus de temps à regarder le premier essai désynchronisé de la phase test que le dernier essai synchronisé de la phase d'habituation $(\mathrm{F}(1,33)=5,74 ; p=0,02)$. Toutefois, on observe une interaction entre le groupe d'âge et les essais, les 


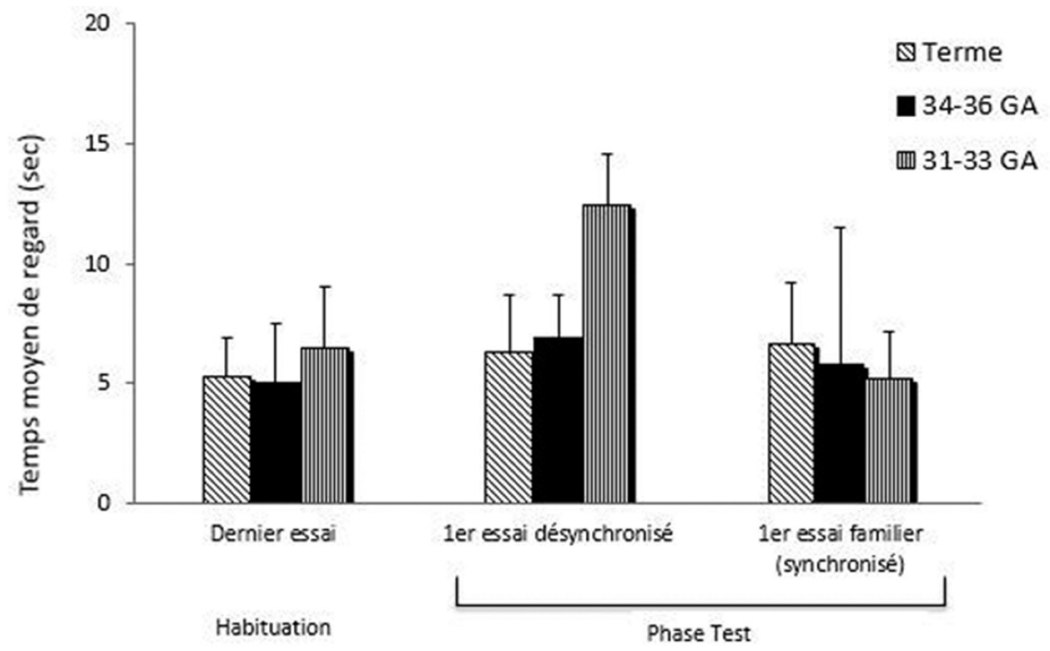

Figure 1.

Temps de regard moyen du dernier essai d'habituation et des deux premiers essais de la phase test (essais désynchronisé et synchronisé) en fonction du groupe d'âge gestationnel.

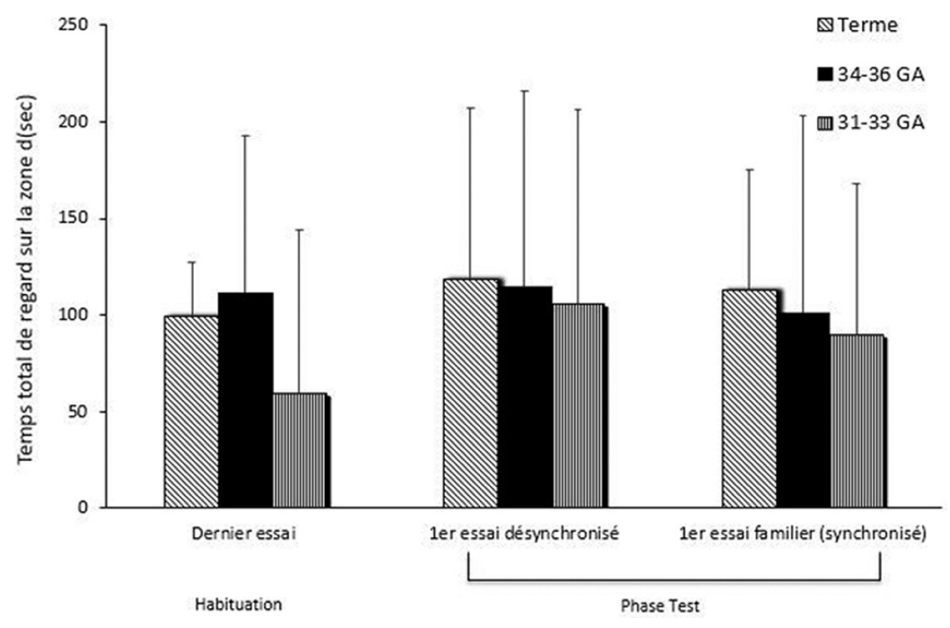

Figure 2.

Temps total de regard sur la zone d pendant le dernier essai d'habituation et des deux premiers essais de la phase test (essais désynchronisé et synchronisé) en fonction du groupe d'âge gestationnel.

bébés de 31-33 sem AG ayant un temps d'exploration de la vidéo désynchronisée particulièrement long, même comparé au premier essai synchronisé de la phase test $(\mathrm{F}(4,66)=2,89 ; p=0,03$; post-hoc Tukey, $p=0,01)$. 


\section{Exploration visuelle}

Les bébés prématurés regardent moins la zone «balle » que les bébés nés à terme $(F(2,23)=25,14, p=0,0001)$. En d'autres termes, leur regard est plus dispersé sur l'écran. Enfin, l'analyse des temps de regards sur la zone d, zone où le son est entendu en décalé dans la scène désynchronisée (fig. 2) montre que les bébés, quel que soit le groupe d'âge, regardent plus cette zone lors de la scène désynchronisée $(F(2,31)=4,17, p=0,02)$. Ce résultat traduit une attention supplémentaire pouvant témoigner de leur surprise d'entendre un son à un moment inhabituel.

\section{Discussion}

Les résultats de cette étude démontrent que la perception de la désynchronie audio-visuelle est présente à 4 mois quel que soit l'âge du terme. Ces travaux vont dans le sens des recherches menées par Lewkowicz (1992) pour les bébés nés à terme, mais contredisent les résultats de Pickens et al. (1994) concernant les bébés prématurés. Ceci se traduit par une augmentation du temps de regard lors du premier essai désynchronisé et une exploration visuelle plus importante de la zone durant laquelle le son décalé apparaît. Ce dernier élément signe une attention visuelle présente même chez les enfants nés prématurément, mise en évidence par le système de traqueur oculaire

Nous pouvons cependant observer une différence au niveau de l'exploration visuelle selon l'âge gestationnel. Les enfants prématurés âgés de 31-33 sem AG mettent plus de temps à s'habituer, leur exploration visuelle est plus dispersée sur l'écran et donc moins centrée sur le trajet de la balle. Ces résultats seraient en faveur de l'hypothèse d'une hypervigilance observée chez les enfants prématurés : ils sont capables d'intégrer des informations bimodales en s'appuyant sur la synchronie temporelle mais auraient des difficultés à inhiber de potentiels distracteurs (Bullinger \& Goubet, 1999).

Il s'agira maintenant d'évaluer plus précisément le rôle et les conséquences de l'expérience extra-utérine de l'enfant né prématurément.

\section{RÉFÉRENCES}

Bloch, H., Lequien, P., \& Provasi, J. (2003). L'enfant prématuré. Paris : Aramnd Colin Bullinger, A., \& Goubet, N. (1999). Le bébé prématuré, acteur de son développement. Enfance, 52(1), 27-32. http://doi.org/10.3406/enfan.1999.3127

Feldman, R., \& Eidelman, A. (2007). Maternal postpartum behavior and the emergence of infant-mother and infant-father synchrony in preterm and full-term infants: The role of neonatal vagal tone. Developmental Psychobiology, 49, 290-302. http://doi.org/10.1002/dev

Lewkowicz, D. J. (1992). Infants' response to temporally based intersensory equivalence: The effect of synchronous sounds on visual preferences for moving stimuli. Infant Behavior and Development, 15(3), 297-324. http://doi.org/10.1016/01636383(92)80002-C 
Mellier, D. (2017). L'enfant prématuré: développement et soins psychologiques. In Miljkovitch, R., Morange-Majoux, F., \& Sander, E. (Eds.), Traité de psychologie du développement. Paris : Masson. pp. 1-36

Pickens, J., Field, T., Nawrocki, T., Martinez, A., Soutullo, D., \& Gonzalez, J. (1994). Full-term and preterm infants' perception of face-voice synchrony. Infant Behavior and Development, 17(4), 447-455. http://doi.org/ http://dx.doi.org/10.1016/0163-6383(94)90036-1 